\title{
Neutron-skin effects in isobaric yield ratio for mirror nuclei in statistical abrasion-ablation model
}

\author{
Chun-Wang $\mathrm{Ma}^{1}$ 囷 Hui-Ling Wei ${ }^{1}$, and Yu-Gang Ma讨 \\ 1 Institute of Particle and Nuclear Physics, Henan Normal University, Xinxiang 453007, China \\ 2 Shanghai Institute of Applied Physics, Chinese Academy of Sciences, Shanghai 201800, China
}

(Dated: January 15, 2018)

\begin{abstract}
Background: The isobaric yield ratio for mirror nuclei $[\operatorname{IYR}(\mathrm{m})]$ in heavy-ion collisions, which is assumed to depend linearly on $x=2(Z-1) / A^{1 / 3}$ of a fragment, is applied to study some coefficients of the energy terms in the binding energy, as well as the difference between the chemical potentials of a neutron and proton. It is found that the $\operatorname{IYR}(\mathrm{m})$ has a systematic dependence on the reaction, which has been explained as the volume and/or the isospin effects in previous studies. However, neither the volume nor the isospin effects can fully interpret the data.

Purpose: We suppose that the IYR $(\mathrm{m})$ depends on the neutron-skin thickness $\left(\delta_{n p}\right)$ of the projectile, and check the idea of whether the neutron-skin thickness effects can fully explain the systematic dependence of the IYR(m).

Methods: A modified statistical abrasion-ablation model is used to calculate the reactions induced by projectiles of three series: (1) the calcium isotopes from ${ }^{36} \mathrm{Ca}$ to ${ }^{56} \mathrm{Ca}$ as projectiles with different limitations on the impact parameters $\left(b_{\max }\right)$ to show the volume effects according to $b_{\max } ;(2)$ the $A=45$ isobars as the projectiles having different isospins and $\delta_{n p}$; and (3) projectiles having similar $\delta_{n p}$ to show whether the IYR(m) depends on the volume or the isospin of the projectile.

Results: The IYR $(\mathrm{m})$ shows a distribution of a linear part in the small- $x$ fragments, and a nonlinear part in the large- $x$ fragments. The linear part of $\operatorname{IYR}(\mathrm{m})$ is fitted. (1) In the calcium isotopic reactions, the $\operatorname{IYR}(\mathrm{m})$ depends on the isospin or the volume of the projectile, but $\delta_{n p}$ greatly influences the nonlinear part of the IYR $(\mathrm{m})$. The $\operatorname{IYR}(\mathrm{m})$ does not depend on the colliding source in reactions of small $b$ max for the nonneutron-rich projectiles, and does not depend on the collision sources in reactions by the neutron-rich projectiles; (2) In reactions of the $A=45$ isobars, though IYR $(\mathrm{m})$ depends on the isospin of projectile, $\operatorname{IYR}(\mathrm{m})$ shows small dependence on isospin if $\delta_{n p}>0$; (3) In the reactions of projectiles having similar $\delta_{n p}$, the IYR(m) in the small mass fragments show no dependence on the volume and the isospin of the projectile when the mass of the projectile is relatively large. Specially, the dependence of $\operatorname{IYR}(\mathrm{m})$ on the mass of the isospin of the projectile vanishes when $\delta_{n p} \sim 0.02 \mathrm{fm}$.
\end{abstract}

Conclusions: The linear and nonlinear parts of the IYR $(\mathrm{m})$ are governed by the core and the surface (skin) of the projectile, respectively. The neutron-skin effects can well explain the systematic dependence of the IYR(m).

PACS numbers: 25.70.Pq, 21.65.Cd, 25.70.Mn

Keywords: isobaric ratio, finite effects, symmetry energy, neutron skin, isospin effect

\section{INTRODUCTION}

In studying the nuclear symmetry energy, the isobaric methods have attracted much attention recently. The energy terms contributing to the binding energy of a nucleus or fragment, which only depend on the mass number, cancel out in the difference between the binding energies of isobars. This makes the isobaric methods possible to study the retained terms in the mass formula. For example, the symmetry energy of neutron-rich nucleus is studied via the difference between binding energies of isobars [1 3 ]. In models based on free energy, the yield of a fragment is determined by its free energy, the properties of the colliding source, and the temperature [4 6]. The symmetry energy of fragment at finite temperature in heavy-ion collisions (HICs), which has a finite temperature, is also studied using the isobaric yield ratio (IYR) methods. After the work using the IYR to

\footnotetext{
* Email: machunwang@126.com

† Email: ygma@sinap.ac.cn
}

study the ratio of the symmetry energy coefficient to the temperature $\left(a_{\text {sym }} / T\right)$ of a fragment [7], the results using the IYR methods are also discussed using the statistical multifragmentation model [8], the canonical and the grand canonical ensembles methods [9, 18], and freeenergy-based models [4, 5]. Moreover, the IYR methods are also used to study the $a_{\mathrm{sym}} / T$ of neutron-rich fragments [10 13], the formation time of fragments [14, 15], the difference between the chemical potentials of a neutron and proton [16], and the temperature of the heavy fragments [17].

In particular, the IYR for mirror nuclei $[\operatorname{IYR}(m)]$, the volume-, surface-, and symmetry-energy terms contributing to the free energy cancel out. The $\operatorname{IYR}(\mathrm{m})$ can be written as follows [7, 10, 19]:

$$
\operatorname{IYR}(\mathrm{m})=\ln \left(Y_{2} / Y_{1}\right)=\left(\Delta \mu+a_{c} \cdot x\right) / T,
$$

with $Y_{2}$ and $Y_{1}$ being the yields of the $I=1$ and -1 ( $I=N-Z$ is the neutron excess) fragments, respectively; $\Delta \mu=\mu_{n}-\mu_{p}, \mu_{n}$ and $\mu_{p}$ being the chemical potentials of the neutron and proton, respectively. $a_{c}$ is the Coulombenergy coefficient and $T$ is temperature; $\operatorname{IYR}(\mathrm{m})$ depends linearly on $x$, with $x=2(z+1 / 2) / A^{1 / 3}$ ( $x$ is the charge 
number of the $I=-1$ fragment) as in Ref. [18] and $x=2(z-1) / A^{1 / 3}$ in Ref. 7, 1013 since different form of Coulomb energy are adopted, but it is confirmed that the two choices of $x$ introduce a very small difference. The $a_{c} \cdot x$ can be seen as the residue Coulomb interaction (RCI) between the related isobars [18].

Based on Eq. (10), the values of $a_{c} / T$ and $\Delta \mu / T$ can be obtained from the IYR $(\mathrm{m})$. A linear correlation between the $\operatorname{IYR}(\mathrm{m})$ and $(Z / A)_{\text {sys }}$ of the reaction system is used to determine $a_{c} / T$ and $\Delta \mu / T$ [7]. Marini et al. provided a method to figure out the RCI by fitting the difference between IYRs [4]. It has been concluded that the $\operatorname{IYR}(\mathrm{m})$ depends on the volume of the reaction systems using the standard grand-canonical and canonical statistical ensembles (SGC/CSE) theories, which prevents the IYR method from obtaining the actual values from fitting nuclear collision data [18]. But conclusions disagree with the SGC/CSE theories proposed in a modified statistical abrasion-ablation (SAA) model by considering the density difference in the projectile, i.e., the $\operatorname{IYR}(\mathrm{m})$ depends on the isospin of the projectile 19]. At the same time, the $\mathrm{SGC} / \mathrm{CSE}$ is also shown in part disagreement with the experimental results [19]. Due to the contradiction in the SGC/CSE, experimental and SAA results, it is meaningful to investigate the system dependence of $\operatorname{IYR}(\mathrm{m})$ in different reactions.

Believing the importance of density distribution in determining the yields of fragments and the resultant parameters, such as $\Delta \mu$ and $a_{c}$, in this article, we focus on the investigation of the neutron-skin effects in $\operatorname{IYR}(\mathrm{m})$. The SAA model will be used because it can well reproduce the yield of the fragment [20, 21, 25, 27], though the SAA model does not include the complex evolution process like the antisymmetrized molecular dynamics models [20]. The article is organized as follows. The SAA model is briefly introduced in Sec. II. The results and discussion are given in Sec. III] and a summary is presented in Sec. IV]

\section{MODEL DESCRIPTION}

In brief, the SAA model is a two-stages model to that predicts the yield of fragments in reactions above the Fermi energy. The first stage describes the colliding, in which the abraded nucleons and the yield of the hot prefragment are determined. The second stage is the evaporation after which the final fragments are formed. It can well reproduce the yield of fragments and is used in studying the isospin phenomena in HICs 22 27.

In the colliding stage, the nuclei are described to be composed of parallel tubes orienting along the beam direction. The SAA takes independent nucleon-nucleon collisions as the participants in the overlap zone of the projectile and target nuclei and determines the distributions of abraded neutrons and protons. For an infinitesimal tube in the projectile, the transmission probabilities for neutrons (protons) at a given impact parameter $\vec{b}$ are given by,

$$
t_{k}(\vec{s}-\vec{b})=\exp \left\{-\left[\rho_{n}^{T}(\vec{s}-\vec{b}) \sigma_{n k}+\rho_{n}^{P}(\vec{s}-\vec{b}) \sigma_{p k}\right]\right\},
$$

where $\rho^{T}$ is the nuclear-density distribution of the target integrated along the beam direction, the vectors $\vec{s}$ and $\vec{b}$ are defined in the plane perpendicular to the beam, and $\sigma_{k^{\prime} k}$ is the free nucleon-nucleon reaction cross section. At a given $\vec{b}$, the average absorbed mass in the limit of infinitesimal tubes is,

$$
\begin{aligned}
<\Delta A(b)> & =\int d^{2} s \rho_{n}^{T}(\vec{s})\left[1-t_{n}(\vec{s}-\vec{b})\right] \\
& +\int d^{2} s \rho_{p}^{P}(\vec{s})\left[1-t_{p}(\vec{s}-\vec{b})\right] .
\end{aligned}
$$

The $\rho_{n}$ and $\rho_{p}$ distributions are assumed to be the Fermitype,

$$
\rho_{i}(r)=\frac{\rho_{i}^{0}}{1+\exp \left(\frac{r-C_{i}}{t_{i} f_{i} / 4.4}\right)}, \quad i=n, p,
$$

where $\rho_{i}^{0}$ is the normalization constant, $t_{i}$ is the diffuseness parameter, and $C_{i}$ is the radius at half density of the neutron or proton density distribution. $t_{i}$ and $C_{i}$ can be adjusted by $f_{i}$ to change the neutron skins thickness $\left(\delta_{n p}\right)$ of a nucleus 28 33]. $\delta_{n p}$ of a nucleus is defined as the difference between the root-mean-square radii of the neutrons' and protons' density distributions $\left(\delta_{n p}=<r_{n}^{2}>^{1 / 2}-<r_{p}^{2}>^{1 / 2}\right)$. In this work we use the default values of the parameters in Eq. (4), and $\delta_{n p}$ of the nucleus is not strictly set to the predicted experimental or theoretical one due to the fact that the measurement of $\delta_{n p}$ itself is still an open question.

The cross section for a specific isotope (prefragment) can be calculated from

$$
\sigma(\Delta N, \Delta Z)=\int d^{2} b P(\Delta N, b) P(\Delta Z, b),
$$

where $P(\Delta N, b)$ and $P(\Delta Z, b)$ are the probability distributions for the abraded neutrons and protons at a given impact parameter $b$, respectively.

The second stage of the reaction in SAA is the evaporation of the excited prefragment 23], which is described by a conventional statistical model under the assumption of thermal equilibrium. The excitation energy of the projectile spectator is estimated by a simple relation of $E^{*}=13.3<A(b)>\mathrm{MeV}$, where 13.3 is the mean excitation energy due to an abraded nucleon from the initial projectile [23]. After the evaporation, the isotopic yield (final fragment) comparable to the experimental result can be obtained. It is shown that the deexcitation or decay descriptions (GEMINI, SMM, SIMON, etc.) greatly influence the yield of the fragment, and the parameters based on the yield [7, [8, 13, 20]. Specially, the prefragment and final fragment in the ${ }^{60} \mathrm{Ni}+{ }^{12} \mathrm{C}$ reaction, the resultant IYR and $a_{\text {Sym }} / T$ of the prefragment and the final fragment are analyzed, which show that the deexciation process affects the results greatly [13]. Since the 
decay mode in SAA can well reproduce the yield of the final fragment, which will be used in the analysis, the effect of different decay mechanism will not be discussed in this work.

\section{RESULTS AND DISCUSSION}

The isospin $\left[I^{\prime}=(N-Z) / A\right]$, volume (or mass) and neutron-skin effects in IYR $(\mathrm{m})$ will be studied in the SAA model. The reactions induced by projectiles of three series will be calculated: (1) the isotopic projectiles to study the isospin and volume effects in $\operatorname{IYR}(\mathrm{m})$, at the same time, different limitations on the maximum of impact parameters will be used to study the volume dependence of $\operatorname{IYR}(\mathrm{m}) ;(2)$ the isobaric projectiles to study the isospin and neutron-skin effects in $\operatorname{IYR}(\mathrm{m})$; and (3) projectiles having similar neutron-skin thickness to study the volume and isospin effects in $\operatorname{IYR}(m)$.

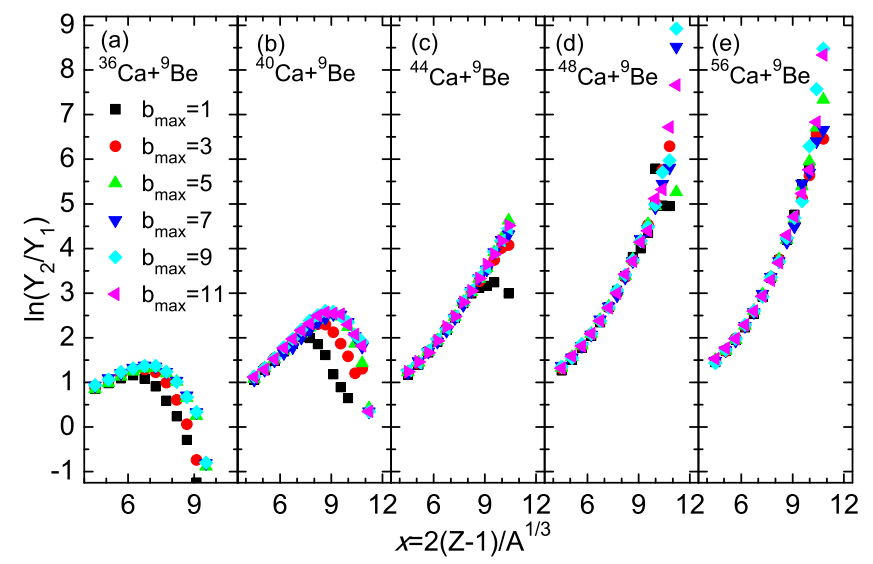

FIG. 1. (Color online) The $\operatorname{IYR}(\mathrm{m})$ in the $140 \mathrm{~A} \mathrm{MeV}$ $36,40,44,48,56 \mathrm{Ca}+{ }^{9} \mathrm{Be}$ reactions with different limitations of maximum impact parameters $\left(b_{\max }\right)$ calculated using the SAA model. $B \max$ is changed from 1 to $11 \mathrm{fm}$ in the step of $2 \mathrm{fm}$.

First, the $140 \mathrm{~A} \mathrm{MeV}{ }^{36,40,44,48,56} \mathrm{Ca}+{ }^{9}$ Be reactions are calculated to study the isospin dependence of the $\operatorname{IYR}(\mathrm{m})$ in the isotopic projectiles. For the projectile from ${ }^{36} \mathrm{Ca}$ to ${ }^{56} \mathrm{Ca}, \delta_{n p}$ are $-0.117,-0.05,0.005,0.053$, and $0.129 \mathrm{fm}$, respectively, and $I^{\prime}$ changes from -0.1 to 0.4. Considering the multiple sources collisions which have different volumes according to the impact parameters, the volume dependence of the $\operatorname{IYR}(\mathrm{m})$ should be manifested. The limitations on the maximum of impact parameter $\left(b_{\max }\right)$ is varied from $1 \mathrm{fm}$ to $11 \mathrm{fm}$ in the step of $2 \mathrm{fm}$ in the calculation. In Fig. 1, the $\operatorname{IYR}(\mathrm{m})$ in these reactions using different $b_{\max }$ are plotted. The IYR $(\mathrm{m})$ shows the distribution as a linear increasing part plus a nonlinear part as $x$ increases. Though the $\operatorname{IYR}(\mathrm{m})$ in the ${ }^{36,40} \mathrm{Ca}$ reactions are easily influenced by $b_{\max }$, the linear part of $\operatorname{IYR}(\mathrm{m})$ changes very little. The IYR $(\mathrm{m})$ in the neutron-rich ${ }^{48,56} \mathrm{Ca}$ reactions are scarcely affected by $b_{\max }$. The $\operatorname{IYR}(\mathrm{m})$ in the ${ }^{44} \mathrm{Ca}$ reactions of $b_{\max }=1 \mathrm{fm}$ only shows very little difference to those of the other $b_{\max }$. In the reactions of neutron-rich projectiles, the volume dependence of the IYR $(\mathrm{m})$ disappears when $b \max$ changes.

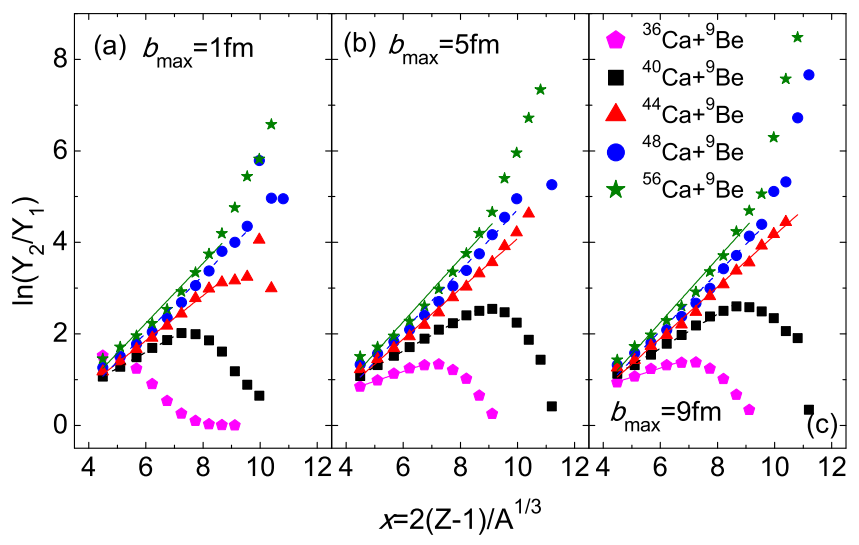

FIG. 2. (Color online) The $\operatorname{IYR}(\mathrm{m})$ in the calculated $140 \mathrm{~A}$ $\mathrm{MeV}{ }^{36,40,44,48,56} \mathrm{Ca}+{ }^{9} \mathrm{Be}$ reactions with limitations of $b_{\max }=1,5$, and 9 , respectively. The lines are the linear fitting results of $\operatorname{IYR}(\mathrm{m})$.

In Fig. 2, the results in Fig. 1 1 are re-plotted according to $b_{\max }$. The $\operatorname{IYR}(\mathrm{m})$ changes according to the colliding volumes in the isotopic reactions. It is clearly shown that, in the large- $x$ fragments, the trend of the nonlinear part of IYR(m) changes from decreasing to increasing as $x$ increases. It can not be definitely explained whether the IYR $(m)$ fully depends on the isospin or the volume of the projectile as has been discussed in Ref. 19]. Generally, the structure of a nucleus can be considered as a core plus a skirt region: in the core region, the density changes little, while in the surface the density decreases quickly. According to Eq. (5), at a specific incident energy, the isotopic yield is mainly determined by the $\rho_{n}$ and $\rho_{p}$ distributions at a specific incident energy. In the surface region, the quick change of density has a great influence on the yield of the fragment in the (semi-)peripheral reactions. In the neutron-rich nucleus, compared to $\rho_{p}$, the relative slow change of $\rho_{n}$ in the surface forms the neutron-skin structure. It should be noted that a neutron-rich nucleus does not guaranty it has a neutron skin since more neutrons are needed to compensate the Coulomb interaction in the large- $Z \mathrm{nu}-$ cleus. It can be assumed that the neutron-skin structure should be more appropriate to explain the phenomena shown in the $\operatorname{IYR}(\mathrm{m})$, i.e., the linear part of $\operatorname{IYR}(\mathrm{m})$ can be explained as the little variation of $\rho_{n}$ and $\rho_{p}$ in the core, and the different trends of the nonlinear part in IYR $(\mathrm{m})$ could be explained as the neutron-skin effect when considering the difference between $\rho_{n}$ and $\rho_{p}$ in the nuclear surface.

To illustrate whether IYR(m) depends on the neutron skin of the projectile, the reactions of some $A_{p}=45$ isobars, i.e., the $140 \mathrm{~A} \mathrm{MeV}{ }^{45} Z+{ }^{9} \mathrm{Be}$ reactions, are calculated. $Z$ of the selected projectiles are $Z=14$, 


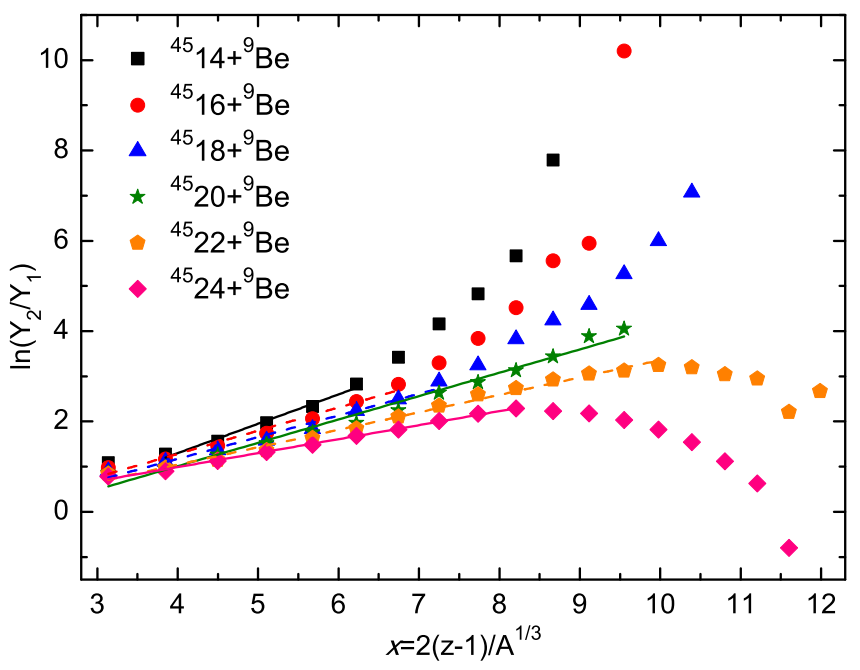

FIG. 3. (Color online) The $\operatorname{IYR}(\mathrm{m})$ in the calculated $140 \mathrm{~A}$ $\mathrm{MeV}{ }^{45} \mathrm{Z}+{ }^{9}$ Be reactions. The projectile changes from $Z=$ 14 to 24 in a step of 2 . The lines are the linear fitting results of $\operatorname{IYR}(\mathrm{m})$.

$16,18,20,22$, and 24 , of which $I^{\prime}$ changes from 0.38 to -0.07 . The $\delta_{n p}$ of the projectiles are $0.179,0.127$, $0.073,0.018,-0.038$, and $-0.096 \mathrm{fm}$, respectively. The $\operatorname{IYR}(\mathrm{m})$ in these reactions are plotted in Fig. 3, which has the similar distribution as in the calcium isotopic reactions. Since the volume of the isobaric projectiles shall be the same, the volume dependence of $\operatorname{IYR}(\mathrm{m})$ can be eliminated in these reactions. The difference among the nonlinear part of $\operatorname{IYR}(\mathrm{m})$ should be explained as the neutron-skin effects.

Furthermore, to show how the neutron-skin affects the $\operatorname{IYR}(\mathrm{m})$, the reactions induced by the projectiles having similar $\delta_{n p}(\sim 0.02,0.06,0.09,0.13$, and $0.18 \mathrm{fm})$ are calculated. The selected $\delta_{n p} \sim 0.02 \mathrm{fm}$ projectiles are ${ }^{25} 11$, ${ }^{35} 15,{ }^{45} 20,{ }^{65} 29$, and ${ }^{85} 38$; the $\delta_{n p} \sim 0.06 \mathrm{fm}$ projectiles are ${ }^{32} 14,{ }^{42} 17,{ }^{52} 21,{ }^{62} 25$, and ${ }^{70} 30$; the $\delta_{n p} \sim 0.09 \mathrm{fm}$ projectiles are ${ }^{24} 9,{ }^{34} 13,{ }^{44} 17,{ }^{54} 21,{ }^{64} 25$ and ${ }^{74} 29$; the $\delta_{n p} \sim 0.13 \mathrm{fm}$ projectiles are ${ }^{26} 9,{ }^{37} 13,{ }^{48} 17,{ }^{66} 24,{ }^{77} 28$ and ${ }^{86} 31$; the $\delta_{n p} \sim 0.18 \mathrm{fm}$ projectiles are ${ }^{30} 9,{ }^{40} 12,{ }^{50} 16$, ${ }^{60} 19,{ }^{70} 22$ and ${ }^{80} 26$, which covers a large range of mass from 25 to 86 , and $I^{\prime}$ from 0.1 to 0.4 . The target nucleus is ${ }^{9} \mathrm{Be}$ and the incident energy is $140 \mathrm{~A} \mathrm{MeV}$. In Fig. 4 the $\operatorname{IYR}(\mathrm{m})$ in reactions of the $\delta_{n p} \sim 0.02,0.09$ and $0.18 \mathrm{fm}$ projectiles are plotted. In Fig. 廿(a), the $\operatorname{IYR}(\mathrm{m})$ in the $\delta_{n p} \sim 0.02 \mathrm{fm}$ projectile reactions overlap, and a quite good linear correlation between $\operatorname{IYR}(\mathrm{m})$ and $x$ is shown in each reaction. No isospin and volume dependence of $\operatorname{IYR}(\mathrm{m})$ is shown. From Figs. 4(b) to 4 (c), when the projectile becomes more neutron-rich, the $\operatorname{IYR}(\mathrm{m})$ shows a quick increase in fragments having large $x$, being the same as those shown in the calcium isotopes and the ${ }^{45} \mathrm{Z}$-induced reactions. In these neutronrich-projectile-induced reactions, the $\operatorname{IYR}(\mathrm{m})$ is greatly influenced by $A_{p}$ when $A_{p}$ is relatively small. When $A_{p}$ is relative large, the $\operatorname{IYR}(\mathrm{m})$ overlap in a large range

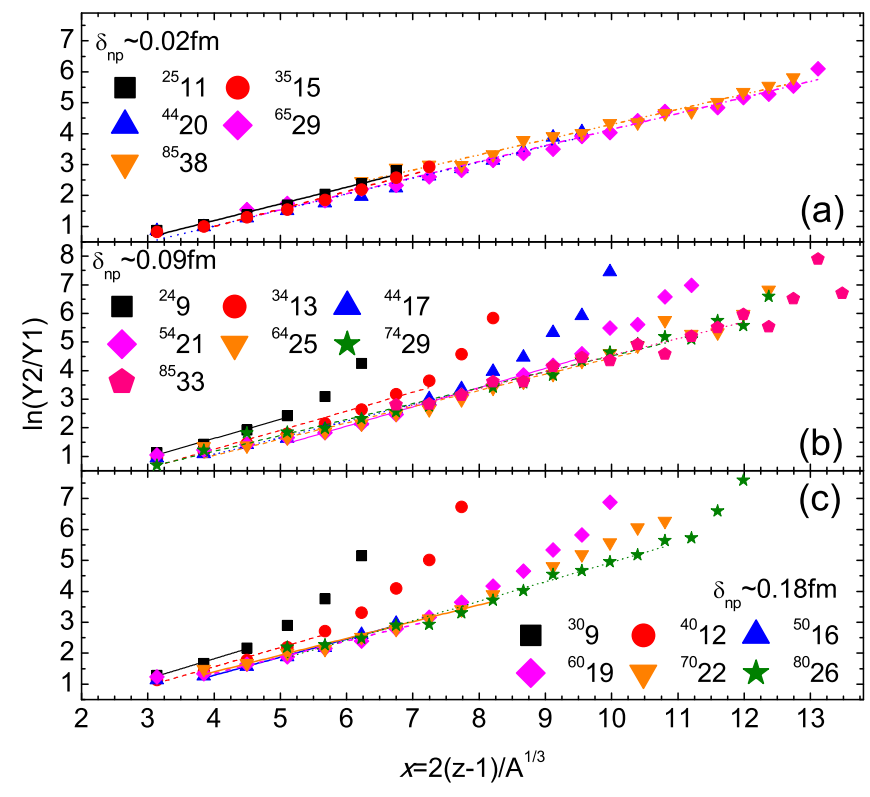

FIG. 4. (Color online) The IYR(m) in the calculated $140 \mathrm{~A}$ $\mathrm{MeV}{ }^{A} \mathrm{Z}+{ }^{9}$ Be reactions induced by projectiles having similar neutron-skin thickness $\left(\delta_{n p}\right) . A$ and $Z$ are the charge and mass numbers of the projectile. In (a), (b) and (c), the reactions are for projectiles having $\delta_{n p} \approx 0.02,0.09$, and $0.18 \mathrm{fm}$, respectively. The lines represent the linear fitting results of the $\operatorname{IYR}(\mathrm{m})$.

of $x$ (for example, $A_{p}>34$ when $\delta_{n p} \sim 0.09 \mathrm{fm}$, and $A_{p}>50$ when $\left.\delta_{n p} \sim 0.18 \mathrm{fm}\right)$. It can be concluded that the $\operatorname{IYR}(\mathrm{m})$ depend on $A_{p}$ very little in the neutron-rich projectile induced reactions when $A_{p}$ is large, which can be explained as that these projectiles have relative large cores in which $\rho_{n}$ and $\rho_{p}$ change very little. The isospin dependence of the $\operatorname{IYR}(\mathrm{m})$ in these reactions will be discussed later.

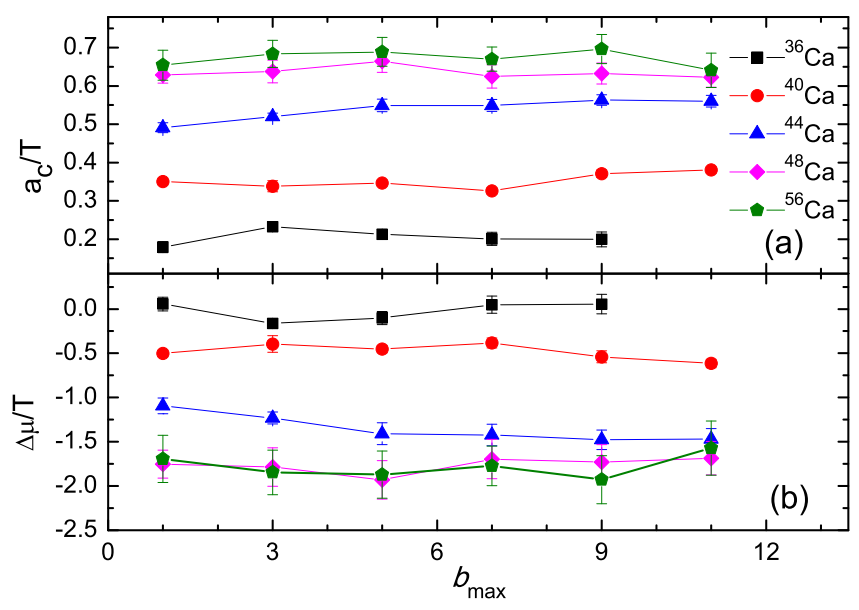

FIG. 5. (Color online) The $a_{c} / T$ and $\Delta \mu / T$ from the fitting result of the linear part of the $\operatorname{IYR}(\mathrm{m})$ in the calcium isotopic reactions which are plotted in Fig. 1. The $x$ axis represents the limitation of the maximum impact parameter $(b \max )$. 
To show the neutron-skin effects in the $\operatorname{IYR}(\mathrm{m})$ more clearly, the linear part of the IYR(m) is fitted using a linear function. In Figs. 5 (a) and 5 (b), the $a_{c} / T$ and $\Delta \mu / T$ determined from the calcium isotopic reactions are plotted, respectively. The $a_{c} / T(\Delta \mu / T)$ is found to increase (decrease) when the projectile becomes more neutronrich, but $a_{c} / T$ and $\Delta \mu / T$ varies very slowly as $b_{\max }$ becomes larger. It can be concluded that in central collisions where the skin has less influence on the yields of fragments, the $\operatorname{IYR}(\mathrm{m})$ does not depend on the colliding volume, whether the projectile is neutron-rich or not.

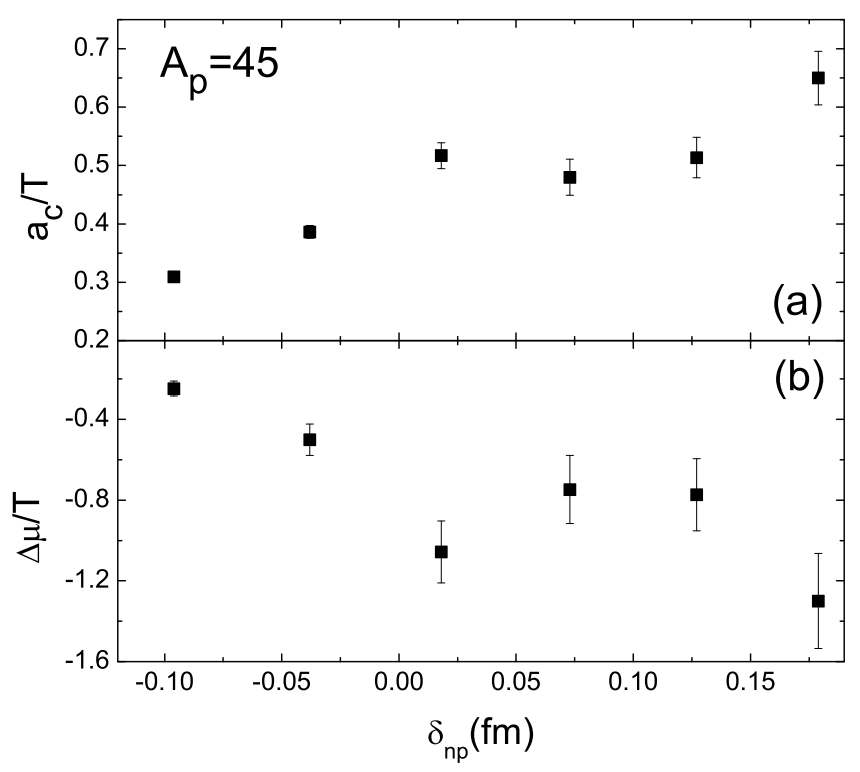

FIG. 6. (Color online) The $a_{c} / T$ and $\Delta \mu / T$ from the fitting result of the linear part of $\operatorname{IYR}(\mathrm{m})$ in the isobaric reactions plotted in Fig. 3 The $x$ axis represents the neutron-skin thickness of $A_{p}=45$ isobars.

The $a_{c} / T$ and $\Delta \mu / T$ determined from the $\operatorname{IYR}(\mathrm{m})$ in the isobaric-projectiles-induced reactions are plotted as a function of $\delta_{n p}$ in Figs. 6(a) and 6(b), respectively. The $a_{c} / T$ increases as $\delta_{n p}$ of the projectile becomes larger, but $a_{c} / T$ only shows little difference when $\delta_{n p}>0$. The $a_{c} / T$ and $\Delta \mu / T$ determined from the $\operatorname{IYR}(\mathrm{m})$ in the reactions of the similar $\delta_{n p}$ projectiles are plotted in Figs. 77(a) and $7(\mathrm{~b})$, respectively. Both the values of $a_{c} / T(\Delta \mu / T)$ are similar (especially when $A_{p}>40$ ). Based on the results in Figs. 6 and 7, it can be concluded that, in the reaction of a projectile having $\delta_{n p}>0$, if the $\operatorname{IYR}(\mathrm{m})$ of fragments vulnerable to the neutron-skin effect is not considered, the volume dependence of the IYR(m) disappears when $A_{p}$ is relatively large. In the reaction induced by a projectile having similar $\delta_{n p}$, the $\operatorname{IYR}(\mathrm{m})$ does not depend on the colliding volumes in the central collisions. Thus in these reactions, the $a_{c} / T$ and $\Delta \mu / T$ determined are not influenced by the mass or volume of the projectile. In particular, in the $\delta_{n p} \sim 0$ projectiles, in which the neutrons and protons distributions are almost equal, the $\operatorname{IYR}(\mathrm{m})$, and the resultant $a_{c} / T$ and $\Delta \mu / T$ are not affected by the mass or volume of the projectile.

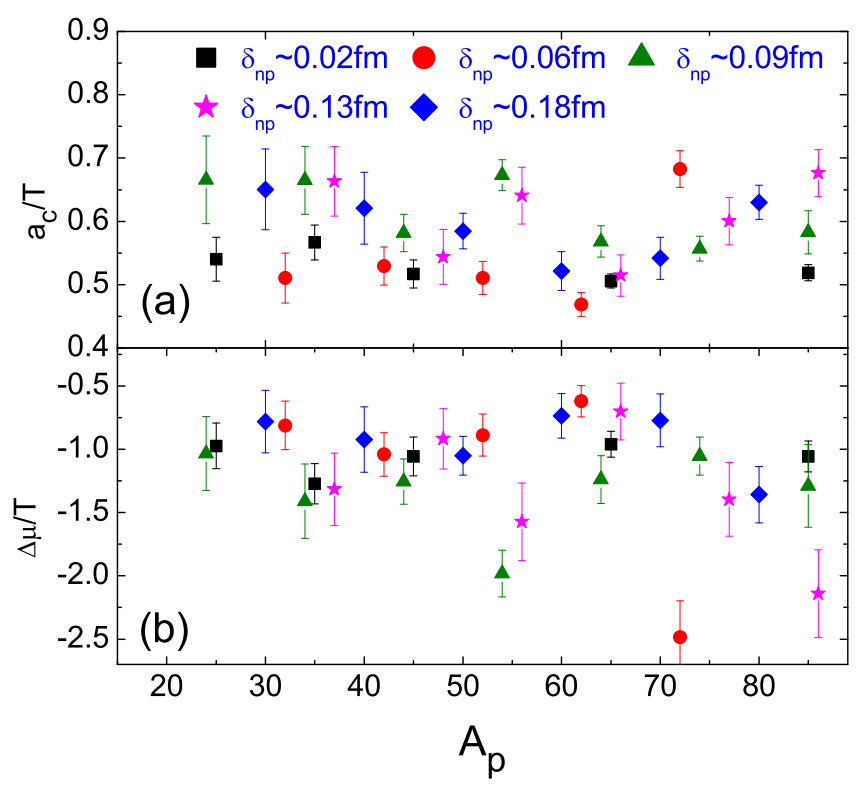

FIG. 7. $a_{c} / T$ and $\Delta \mu / T$ from the fitting result of the linear part of the $\operatorname{IYR}(\mathrm{m})$ in the reactions induced by projectiles having $\delta_{n p} \sim 0.02,0.06,0.09,0.13$, and $0.18 \mathrm{fm}$. Some $\operatorname{IYR}(\mathrm{m})$ of these reactions are plotted in Fig. 4 The $x$ axis represents the mass of the projectile $\left(A_{p}\right)$.

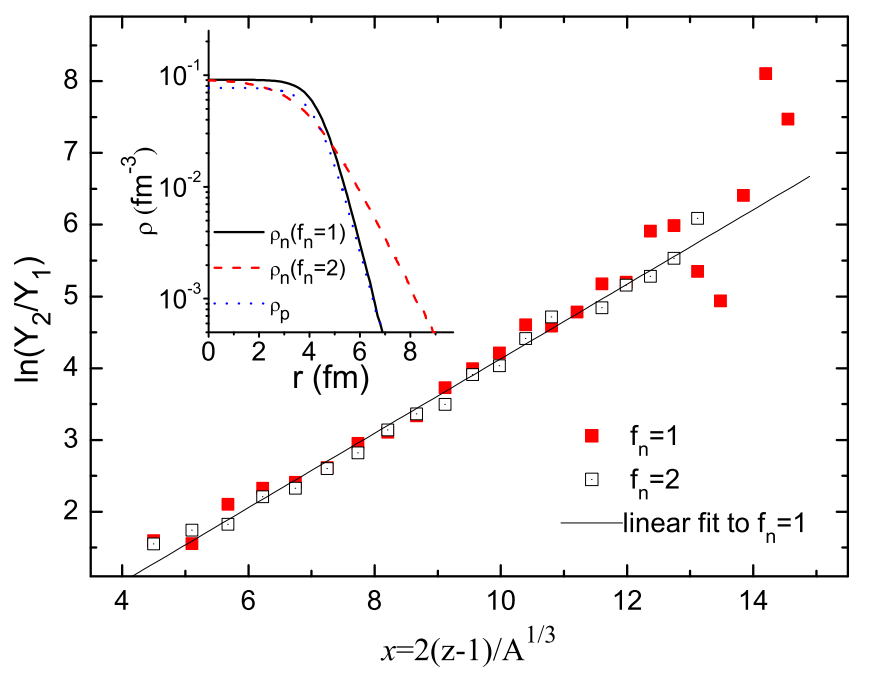

FIG. 8. (Color online) The IYR $(\mathrm{m})$ in the calculated $140 \mathrm{~A}$ $\mathrm{MeV}{ }^{65} 29+{ }^{9}$ Be reactions. $\delta_{n p}$ of ${ }^{65} 29$ is adjusted by changing $f_{n}$ in Eq. (4). The open and solid squares represent the results of $f_{n}=1$ and 2 , respectively. The line is the linear fitting result to the $\operatorname{IYR}(\mathrm{m})$ of $f_{n}=1$. In the inserted figure, the lines represent $\rho_{n}$ according to $f_{n}=1$ (solid), 2 (dashed), and $\rho_{p}$ (dotted) of ${ }^{65} 29$ according to Eq. (4).

It is interesting to note how $\operatorname{IYR}(\mathrm{m})$ change if the neutron-skin thickness is adjusted. By changing the value of $f_{i}$ in Eq. (4), the diffuseness in $\rho_{n}$, at the same time $\delta_{n p}$ can be changed. For the ${ }^{A} Z={ }^{65} 29$ nucleus, $\delta_{n p}=0.02$ 
and $0.85 \mathrm{fm}$ when $f_{n}=1$ and 2 , respectively. In Fig. 8, the $\operatorname{IYR}(\mathrm{m})$ according to $f_{n}=1$ and 2 in the reactions are plotted, and the $\rho_{n}$ and $\rho_{p}$ distributions are plotted in the inserted figure. Compared to the result of $f_{n}=1$, more fragments of large mass are produced in the result of $f_{n}=2$. The $\operatorname{IYR}(\mathrm{m})$ in the $f_{n}=1$ and 2 results almost overlap, but an increase can be found in the isobars of $x>10$ [the $\operatorname{IYR}(\mathrm{m})$ of $x>12$ show the accelerating increase trend]. It can be concluded that the change of density distribution does change the $\operatorname{IYR}(\mathrm{m})$ distribution.

Finally, we discuss the isospin dependence of the $\operatorname{IYR}(\mathrm{m})$. The nonlinear part of $\operatorname{IYR}(\mathrm{m})$ is omitted in the determination of the $a_{c} / T$ and $\Delta \mu / T$. It was noted previously that the nonlinear part was due to the skin effects in the $\operatorname{IYR}(\mathrm{m})$. An equivalent "isospin" can be defined as $\left(\rho_{n}-\rho_{p}\right) /\left(\rho_{n}+\rho_{p}\right)$. For neutron-rich (or proton-rich) projectiles, in some sense, the "isospin" changes quickly in the surface region due to the fast change of $\rho_{n}$ and $\rho_{p}$; while the "isospin" keeps constant in the core region. In Figs. 9(a) and 9(b), the $a_{c} / T$ and $\Delta \mu / T$ in Fig. 7 are re-plotted as a function of the isospin of the projectile. From $I^{\prime}=0.1$ to 0.4 , the $a_{c} / T$ shows a small difference, and even less variation in $\Delta \mu / T$ is found. It is concluded that if $a_{c} / T$ and $\Delta \mu / T$ determined from the linear part of $\operatorname{IYR}(\mathrm{m})$, the isospin effects are also very small. Thus the neutron-skin effects can well explain the systematic phenomena shown in $\operatorname{IYR}(\mathrm{m})$.

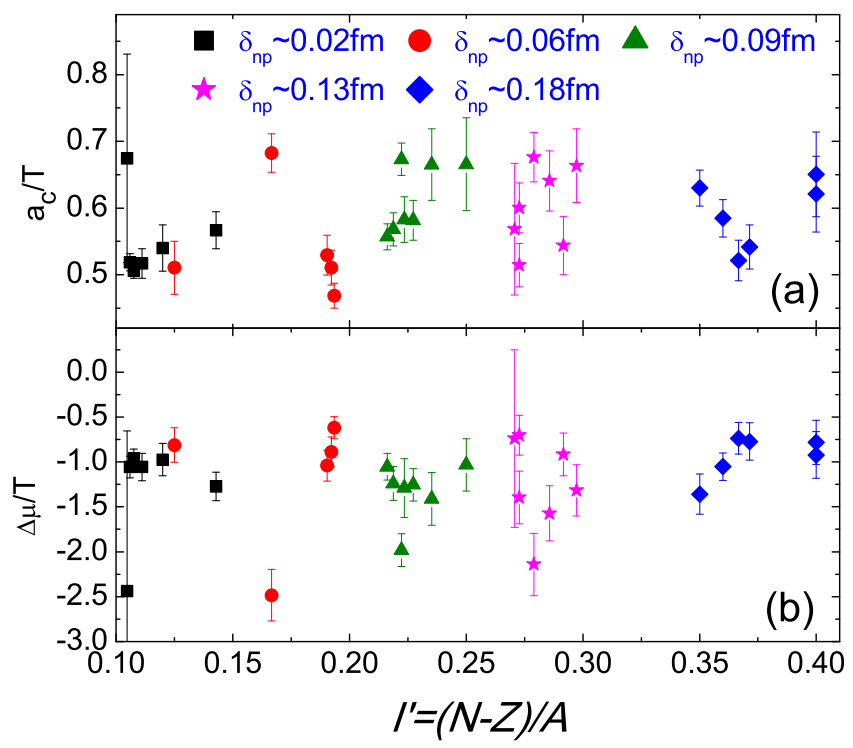

FIG. 9. (Color online) The $a_{c} / T$ and $\Delta \mu / T$ from the fitting of the linear part of IYR $(\mathrm{m})$ in the reactions of projectiles with $\delta_{n p} \sim 0.02,0.06,0.09,0.13$, and $0.18 \mathrm{fm}$. The $x$ axis represents the isospin parameter $\left[I^{\prime}=(N-Z) / A\right]$ of the projectile.

\section{SUMMARY}

In summary, we focus on the interpretation of the systematic dependence of $\operatorname{IYR}(\mathrm{m})$ and the extracted parameters from them. The previously proposed isospin or volume dependence of IYR $(\mathrm{m})$ did not fully explain the phenomena shown in the experimental results, or the theoretical results of the SAA and the SGC/CSE models. Considering the density effects in the fragment production, it is assumed that $\delta_{n p}$ of the projectile affects the $\operatorname{IYR}(\mathrm{m})$. Using the SAA model, which considers the density difference of a proton and neutron conveniently, the reactions induced by three series of projectiles are calculated: (1) the calcium isotopes from ${ }^{36} \mathrm{Ca}$ to ${ }^{56} \mathrm{Ca}$ with different limitations of $b_{\max }$; $(2)$ the $A=45$ isobaric projectile of which $Z$ varies from 14 to 24 ; and (3) the projectiles having similar $\delta_{n p}$. Generally, the $\operatorname{IYR}(\mathrm{m})$ in the calculated reaction shows the distribution of a linear part in the small- $x$ fragments and a nonlinear part in the large- $x$ fragments except in the reactions of $\delta_{n p} \sim 0$ projectiles. The linear part of $\operatorname{IYR}(\mathrm{m})$ is explained as the core effects of the projectile, and the nonlinear part of $\operatorname{IYR}(\mathrm{m})$ is assumed to be governed by the skin of the projectile. In the calcium isotopes induced reactions, it is shown that $\operatorname{IYR}(\mathrm{m})$ does not depend on the volume of the colliding source in the central collisions, whether the projectile is neutron-rich or not. In the isobaric reactions, the $\operatorname{IYR}(\mathrm{m})$ is found to be greatly influenced by $\delta_{n p}$ of the projectile, but when the projectile has a relatively large $\delta_{n p}$, the $\operatorname{IYR}(\mathrm{m})$ depends on the isospin very little. If the $\delta_{n p}$ of the projectile is similar, IYR $(\mathrm{m})$ does not depend on the mass or volume of the projectile when its mass is relatively large. From the calculated results, it can be concluded that when $\rho_{n}$ and $\rho_{p}$ change very little in the core, or $\rho_{n}$ and $\rho_{p}$ in the entire projectile are similar (such as the $\delta_{n p} \sim 0.02 \mathrm{fm}$ projectiles in which $\rho_{n}$ and $\rho_{p}$ can be assumed to be the same), both the isospin and volume dependence of $\operatorname{IYR}(\mathrm{m})$ disappears. It can be concluded that the system dependence of $\operatorname{IYR}(\mathrm{m})$ shown in the SAA and experimental data shall be the neutron-skin effects, and neither the isospin nor the volume dependence of $\operatorname{IYR}(\mathrm{m})$ can completely explain this dependence. The finite effects suggested in the SGC/CSE results are inadequate to explain the experimental results, and also disagree with the SAA results.

At last, we comment on the $a_{c} / T$ and $\Delta \mu / T$ in the isotopic reactions, which show a dependence on the mass of the projectiles. Due to the values of $a_{c}, \Delta \mu$ and $T$ are difficult to separate in the free-energy models; they influence each other in the fitting. $\Delta \mu$ reflects the properties of the projectile, which can be assumed to increase when the projectile becomes more neutron-rich. In a canonical thermodynamic (CTM) model, a temperature profile of impact parameter $(b)$, which decreases quickly as $b$ increases, is introduced to improve the prediction of the fragment yield [34]. Due the low $\rho_{n}$ and $\rho_{p}$ in the surface (which corresponds to the peripheral collisions), the abraded nucleons are also less than those in the central 
collisions, which can also result in the relatively low temperature 16, 17, 34]. In this sense, the low temperature in the peripheral collisions is one result of the low density [16, 17]. The increasing $a_{c} / T$ in the large- $x$ fragments can also be explained as the density effects since the surface regions of the projectile (target) govern the peripheral collisions. Due to the complexity of the temperature dependence on the impact parameters and densities, and the possible dependence of $a_{c}$ on the density [4, 5], we can not know exactly what the actual value of $a_{c}$ is. It was proposed that the RCI between the isobars can be determined by some approximations as in Refs. [4, 7, 35], and it was shown that, though the Coulomb term was retained in determining the symmetry-energy coefficient of the neutron-rich fragment in the IYR method [7, 11 13], the RCI between the isobars in the difference of IYRs was negligible [4, 35].

\section{ACKNOWLEDGMENTS}

This work is supported by the National Natural Science Foundation of China (Grants No. 10905017 and 11035009), the Knowledge Innovation Project of the Chinese Academy of Sciences under Grant No. KJCX2EW-N01, the Program for Science \& Technology Innovation Talents in Universities of Henan Province (13HASTIT046), and the Young Teacher Project in Henan Normal University.
[1] J. Jänecke, and T. W. O'Donnell, Nucl. Phys. A 781, 317 (2007).

[2] H. Mei et al., J. Phys. G: Nucl. Part. Phys. 39, 015107 (2012).

[3] C. W. Ma et al., Chin. Phys. Lett. 29, 092101 (2012).

[4] P. Marini et al., Phys. Rev. C 85, 034617 (2012).

[5] M. Huang et al., Phys. Rev. C 81, 044618 (2010).

[6] A. S. Hirsch et al., Phys. Rev. C 29, 508 (1984).

[7] M. Huang et al., Phys. Rev. C 81, 044620 (2010).

[8] P. Marini et al., Phys. Rev. C 87, 024603 (2013).

[9] S. Mallik and G. Chaudhuri, Phys. Rev. C 87, 011602(R) (2013).

[10] C. W. Ma, F. Wang, Y. G. Ma and C. Jin, Phys. Rev. C 83, 064620 (2011).

[11] C. W. Ma, J. Pu, H.-L. Wei, S.-S. Wang, H.-L. Song, Eur. Phys. J. A 48, 78 (2012).

[12] C. W. Ma, J. Pu, S.-S. Wang, and H.-L. Wei, Chin. Phys. Lett. 29, 062101 (2012).

[13] C. W. Ma et al., Chin. Phys. C 37, 024102 (2013).

[14] M. Huang et al., Phys. Rev. C 82, 054602 (2010).

[15] M. Huang et al., Nucl. Phys. A 847, 233 (2010)

[16] C. W. Ma, S. S. Wang, Y. L. Zhang, H. L. Wei, Phys. Rev. C 87, 034618 (2013).

[17] C. W. Ma, J. Pu, Y. G. Ma, R. Wada, S. S. Wang, Phys. Rev. C 86, 054611 (2012); C. W. Ma ibid., 88, 014609 (2013);

[18] S. R. Souza and M. B. Tsang, Phys. Rev. C 85, 024603 (2012).

[19] C. W. Ma, S. S. Wang, H. L. Wei, and Y. G. Ma, Chin. Phys. Lett. 30, 052501 (2013).
[20] M. Mocko, M. B. Tsang, D. Lacroix, A. Ono, P. Danielewicz, W. G. Lynch, and R. J. Charity, Phys. Rev. C 78, 024612 (2008).

[21] M. B. Tsang et al., Phys. Rev. C 76, 041302(R) (2007); M. Mocko et al., Europhys. Lett. 79, 12001 (2007).

[22] T. Brohm and K. -H. Schmidt, Nucl. Phys. A 569, 821 (1994).

[23] J. J. Gaimard and K. H. Schmidt, Nucl. Phys. A 531, 709 (1991).

[24] D. Q. Fang et al., Phys. Rev. C 61, 044610 (2000).

[25] H. L. Wei and C. W. Ma, Acta Phys. Sin., 59, 5364 (2010) (in Chinese).

[26] C. W. Ma et al., Phys. Rev. C 79, 034606 (2009).

[27] C. W. Ma, H. L. Wei, J. Y. Wang, and G. J. Liu, Chin. Phys. B 18, 4781 (2009).

[28] C. W. Ma et al. Chin. Phys. B 17, 1216 (2008).

[29] D. Q. Fang, Y. G. Ma, X. Z. Cai, W. D. Tian, and H. W. Wang, Phys. Rev. C 81, 047603 (2010).

[30] C. Ma , Y. Zhang, and C. Jin, Plasma Sci. Technol. 14, 396 (2012).

[31] C. W. Ma, and S. S. Wang, Chin. Phys. C 35, 1017 (2011).

[32] C.-W. Ma, H.-L. Wei, and M. Yu, Phys. Rev. C 82, $057602(2010)$.

[33] C. W. Ma, H. L. Wei, G. J. Liu, and J. Y. Wang, J. Phys. G: Nucl. Part. Phys. 37, 015104 (2010).

[34] S. Mallik, G. Chaudhuri, and S. Das Gupta Phys. Rev. C 84, 054612 (2011).

[35] C. W. Ma, S. S. Wang, Y. L. Zhang, and H. L. Wei, unpublished. 